\title{
Formalin Potentials in the Pathogenic Attenuation of Eimeria tenella based on Oocyst Productions
}

\author{
Resti Devi Anggraini ${ }^{1}$, Epy Muhammad Luqman ${ }^{1}$ and Setya Budhy ${ }^{2}$ \\ ${ }^{I}$ Department of Veterinary Anatomy, Faculty of Veterinary Medicine, Universitas Airlangga, Surabaya-60115, Indonesia \\ ${ }^{2}$ Department of Veterinary Basic Medicine, Faculty of Veterinary Medicine, Universitas Airlangga, Surabaya-60115, Indonesia \\ *Corresponding author's Email: epy-m-1@fkh.unair.ac.id; ORCID: 0000-0002-2821-6319
}

Received: 20 Jan. 2021

Accepted: 03 Mar. 2021

\begin{abstract}
Coccidiosis is a disease found in poultry caused by parasitic protozoa, namely Eimeria tenella (E. tenella), which may lead to high rates of morbidity and mortality. To prevent coccidiosis, vaccination is required to inactivate and attenuate E. tenella protozoa. One of the compounds applied for attenuation is formaldehyde. Formaldehyde reduces the pathogenicity of an organism by creating rigidity in its structure. As a result, the organism cannot inflict disease and has a higher impact on building antibodies although it is still alive. The current research was an experimental study aimed to determine the formalin potential in attenuation of E. tenella pathogenesis in terms of oocyst production. The present study was conducted using the completely randomized design method. A total number of 25 broiler chickens were applied and their feces were tested to observe oocysts production and clinical symptoms. The obtained data would be analyzed by the ANOVA statistical test. The treatment groups presented clinical symptoms of E. tenella infection. The number of oocysts in treatment group I fluctuated from the lowest number which was zero on day five and then increased by day six, seven, and eight and it has reached the peak with the most significant amount of 4,050,460 oocysts on day nine. The treatment group II with the same condition reached its peak with the highest number of 1,363,160 oocysts on day nine. The treatment group III peaked with the most significant number of 618,960 oocysts on day nine. In addition, the treatment IV group attained the apex with the highest number of 719,480 oocysts on day nine. Meanwhile, the treatment V group reached the highest number of 284,200 oocysts on day nine. The difference in formalin concentration affected the amount of E. tenella oocyst production of broiler chickens. Formalin soaking with a concentration of $1.2 \%$ was the most optimal concentration to attenuate E. tenella.
\end{abstract}

Keywords: Broiler chicken, Eimeria tenella, Formalin, Oocyst

\section{INTRODUCTION}

There are some threats to inhibit the sustainability of the poultry sector, one of them is poultry disease (Gussem, 2007; Kadhim, 2014). Poultry can suffer from several diseases due to parasitic protozoa, which may cause high morbidity and mortality. One of the diseases is coccidiosis, caused by a protozoa type and a genus of Eimeria (Dalloul and Lillehoj, 2006; Hafex, 2011; Kadhim, 2014). Eimeria tenella (E. tenella) is one of the Eimeria species that causes plump-formed coccidiosis in chickens. This disease often ravages chickens aged around three weeks. The parasite lives in the cecum epithelium and experiences multiplication in the epithelium. Consequently, it causes weight loss, stunted growth, decreased appetite, pale combs and pallor, tangled fur, diarrhea, reduced meat production, bloody diarrhea, and even death (Fanatico et al., 2007). Coccidiosis is one of the most prevalent diseases, which occurs during the chicken-breeding period (Kadhim, 2014). Various attempts to control coccidiosis have been carried out, although they are not entirely successful. Maintaining clean sanitation is known to break the Eimeria life cycle. This method, nonetheless, cannot completely prevent coccidiosis (Ayed et al., 2012). The use of coccidiostat for a long period has led to problems. The side effect of coccidiostat includes resistance to the coccidiostat, even poisoning. Adding coccidiostat to chicken feed for the long term may cause residues in meat and eggs, which threatens consumers (Srinivasu et al., 2019). Vaccination is an effective and safe alternative to control coccidiosis (Bahrami and Bahrami, 2006; Meeusen et al., 2007). A vaccine is produced through inactivation and attenuation. Pathogenicity attenuation means reducing the ability of an organism to cause disease in infected organisms (Bahrami 
and Bahrami, 2006). If chemicals are utilized for attenuation, that material must produce a minuscule change to stimulate protective immunity in the antigen. One of the compounds used for attenuation is formaldehyde. Formaldehyde reacts with amino and amide groups in proteins, and with amino bound to non-water substances with the essential ingredients of purine nucleic acids and pyrimidines (Tizard, 2004). The chemical forms cross-bonding, and thus provides structural rigidity in the organism. The structural rigidity of these organisms can reduce organism pathogenicity. Even though the organism is alive, it cannot cause any disease. Furthermore, it is highly effective to form antibodies (Tizard, 2004). Based on the explanation above, this research aimed to determine the formalin potential in attenuation of E. tenella pathogenesis in terms of oocyst production.

\section{Ethical approval}

All experimental protocols and procedures were approved by the Institutional Animal Care of Indonesia.

\section{MATERIALS AND METHODS}

The present research was an experimental study using the completely randomized design with an assumption that the experimental animal cages were uniform. The isolations employed were E. tenella oocysts collected from the cecum of domestic chickens, and developed in the Department of Veterinary Parasitology, Universitas Airlangga, Surabaya, Indonesia. Oocysts isolates were field strains known to cause cecal lesions according to Jordan et al. (2011). Sporulation was obtained by constant stirring and also aeration of oocysts for 48 hours at $28^{\circ} \mathrm{C}$.

In the current study, 25 male broiler chickens aged two weeks old, were randomized based on the completely randomized design. Each pen (cage) was provided with water and an individual feeder. Room temperature was maintained at $21^{\circ} \mathrm{C}$. Meanwhile, the experimental design pattern was conducted on five treatment groups with five replications in each group. The present study consisted of five treatment groups. Treatment group I (P-I) was the control group in which oocysts were not immersed in formalin, then about 10,000 oocytes were inoculated. Treatment group II (P-II) involved E. Tenella immersed in formalin with a concentration of $0.15 \%$ for 96 hours, and then inoculated, amounting to 10,000 oocysts per oral. In treatment group II (P-III), E. Tenella was immersed in formalin with a concentration of $0.3 \%$ for 96 hours, and then inoculated, amounting to 10,000 oocysts per oral. Treatment group IV (P-IV) entailed E. tenella immersed in formalin with a concentration of $0.6 \%$ for 96 hours, and then inoculated, amounting to 10,000 oocysts per oral. Finally, in treatment group $\mathrm{V}(\mathrm{P}-\mathrm{V})$, E. tenella was immersed in formalin with a concentration of $1.2 \%$ for 96 hours, and then inoculated, amounting to 10,000 oocysts per oral.

\section{Feces collection}

The chicken feces were collected during 5-12 days after infection, and then they were put into plastic bags and $10 \%$ formalin was added. Then, they were labeled according to the distribution of the treatment groups. Collected chicken feces were stored for a short time before the examination of feces.

\section{Examination and calculation of total oocyst production per day}

The collected feces samples were examined to calculate the amount of oocyst production. The sequence process for calculating the total amount of oocyst production was conducted using the Mc-Master method. The feces samples were weighed as much as 2 grams and then put in a test tube. A total amount of $58 \mathrm{ccs}$ of saturated salt solution was added to the tube containing feces, and then stirred using a stirring pipette until they were homogeneous. Next, the samples were taken using a Pasteur pipette and put into the Mc-Master counting chamber until both chambers became full. Following that, they were calculated under a light microscope with $100 \times$ magnification and were calculated in two Mc-Master counting chambers.

\section{Research variable}

There were three variables in the current research including independent variable, dependent variable, and control variable. The independent variables were formalin concentration and E. tenella immersion time in formalin. The dependent variables were the amount of oocyst production and clinical symptoms. Meanwhile, the control variables in this study were E. tenella oocysts, chicken species, chicken feed, cages, environment, and required tools for the research.

\section{Data analysis}

The data to obtain were the clinical symptoms and oocyst production. The date of clinical symptoms was descriptively presented while the oocysts production data was assessed by Analysis of Variant (ANOVA) in SPSS (version 18) for Windows. If each group were significantly different, a further test with the Least Significant Different 
(LSD) would be carried out. Differences were considered significant when $\mathrm{p}<0.05$.

\section{RESULTS AND DISCUSSION}

\section{Clinical symptoms}

In the control group (P-I) with non-attenuated $E$. tenella infection, the chickens presented clinical symptoms, such as looking weak, loss of appetite, and bloody diarrhea five to six days after the infection. Clinical symptoms in P-II, P-III, and P-IV were relatively similar. In general, the clinical symptoms included looking rather limp chickens, loss of appetite, and watery stool (diarrhea). On the other hand, the P-IV group represented non-significant clinical symptoms $(\mathrm{p}>0.05)$. The chickens in this group had good health, good appetite, and a proper drink. Moreover, the chickens in this group looked more agile than the chickens in the other groups.

\section{Test results of oocyst production}

The results of the E. tenella oocysts production on feces was commenced on day 5 to 12 . The oocysts production on day five was zero for all treatment groups. Therefore, the data included in this section is the production data of oocysts per gram feces on days six to seven after the infection. The examination was performed using the Mc-Master method and was observed under a light microscope with $100 \times$ magnification. Table 1 describes the research data.

\section{Feces samples}

Table 1 indicates that the average amount of oocyst production of the broiler chickens infected by E. tenella in the control group was significantly different in terms of the average result per day, compared to the other groups ( $\mathrm{p}$ $<0.05)$.
Figure 1 demonstrates that the number of oocysts in the P-I group fluctuated from the lowest number of zero on day five and then increased on days six, seven, and eight and reaching the peak with the highest number of $4,050,460$ oocysts on day nine. in the same condition, P-II achieved the summit with the highest number of $1,363,160$ oocysts on day nine. However, P-III hit the culmination with the highest number of 618,960 oocysts on day nine. In addition, treatment IV reached the peak with the highest number of 719,480 oocysts on day nine. Eventually, the P$\mathrm{V}$ group reached the top result with the highest number of 284,200 oocysts on day nine. As a matter of fact, the oocysts productions in the P-I group were always higher during 7-12 days when compared to the other groups. It was due to factor that the treatment in this group was not immersed in formalin. The oocyst production peaked on day nine after infection in the P-I group. In treatment groups with different concentrations of formaldehyde immersion, the oocyst production on day nine was lower than the P-I, without any formaldehyde immersion. After the oocyst production reached the summit on day nine, the amount of oocyst production decreased on the following day.

Based on figure 2, it can be observed that there is a significant difference in the number of oocyst production in each treatment group on the same day $(\mathrm{p}<0.05)$. The control group (P-I) always had the highest number of oocyst production, compared to the treatment groups that $E$. tenella was immersed in formalin with various concentrations. The number of oocysts in P-II was lower than P-I and was higher than P-III. The number of oocytes in P-III was generally higher than in P-IV. Nevertheless, P-III had a lower oocyst production than P-IV on days 9 and 10. The number of oocysts in P-V was lower than PIII and P-IV.

Table 1. Average and standard deviation of oocyst production in feces samples of the broiler chickens infected by Eimeria tenella during 6-12 days after infection.

\begin{tabular}{lccccccc}
\hline Group & Day 6 $\left(\bar{x} \pm S D^{1}\right)$ & Day 7 $(\overline{x \pm S D})$ & Day 8 $(\bar{x} \pm S D)$ & Day 9 $(\bar{x} \pm S D)$ & Day 10 $(\overline{x \pm S D})$ & Day 11 $(\bar{x} \pm S D)$ & Day 12 $(\bar{x} \pm S D)$ \\
\hline Control & $9.4^{\mathrm{a}} \pm 8.013$ & $30.9^{\mathrm{a}} \pm 12.630$ & $355.6^{\mathrm{a}} \pm 215.20$ & $4050.5^{\mathrm{a}} \pm 3095.22$ & $2488^{\mathrm{a}} \pm 1902.34$ & $1341^{\mathrm{a}} \pm 627.393$ & $120.34^{\mathrm{a}} \pm 78.415$ \\
Formalin 0.15\% & $3.54^{\mathrm{ab}} \pm 4.862$ & $19.9^{\mathrm{b}} \pm 4.621$ & $231.04^{\mathrm{ab}} \pm 129.462$ & $1363.2^{\mathrm{b}} \pm 732.344$ & $601.38^{\mathrm{b}} \pm 343.373$ & $238.4^{\mathrm{b}} \pm 136.706$ & $42.240^{\mathrm{b}} \pm 26.988$ \\
Formalin 0.3\% & $2.35^{\mathrm{b}} \pm 3.609$ & $14.6^{\mathrm{b}} \pm 2.897$ & $130.4^{\mathrm{b}} \pm 87.656$ & $618.96^{\mathrm{b}} \pm 324.259$ & $340.74^{\mathrm{b}} \pm 311.717$ & $172.38^{\mathrm{b}} \pm 135.353$ & $35.980^{\mathrm{b}} \pm 35.231$ \\
Formalin 0.6\% & $0.82^{\mathrm{b}} \pm 1.833$ & $3.8^{\mathrm{a}} \pm 2.456$ & $128.87^{\mathrm{b}} \pm 55.665$ & $719.48^{\mathrm{b}} \pm 446.684$ & $354.60^{\mathrm{b}} \pm 116.976$ & $109.4^{\mathrm{b}} \pm 77.105$ & $20.16^{\mathrm{b}} \pm 16.353$ \\
Formalin 1.2\% & $0^{\mathrm{b}}$ & $2.309^{\mathrm{a}} \pm 1.3$ & $40.14^{\mathrm{b}} \pm 33.644$ & $98.644^{\mathrm{b}} \pm 25.718$ & $176.60^{\mathrm{b}} \pm 147.92$ & $116.34^{\mathrm{b}} \pm 75.349$ & $12.38^{\mathrm{b}} \pm 11.47$ \\
\hline
\end{tabular}

Note: Different superscripts in the same columns represent a significant difference $(p<0.05)$ of the treatments per day. ${ }^{1}$ standard deviation 


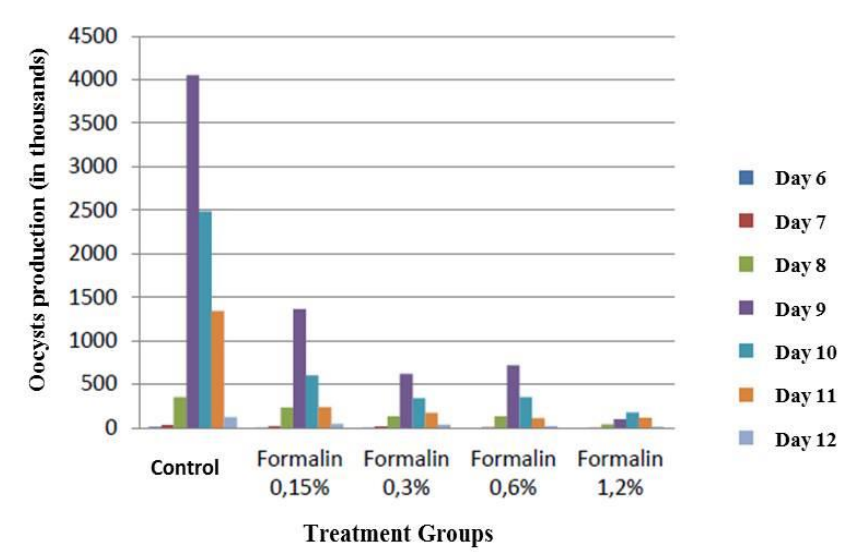

Figure 1. Formalin Potential Graphic of Eimeria tenella pathogenicity which attenuated with different formalin concentrations regarding oocyst production of the broiler chickens infected by Eimeria tenella per day.

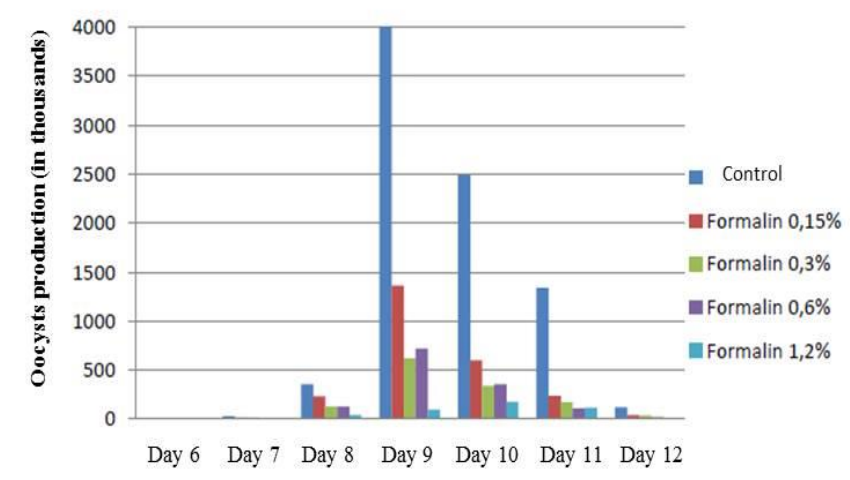

Figure 2. Formalin Potential Graphic of Eimeria tenella pathogenicity which attenuated with different formalin concentrations in terms of oocyst production of the broiler chickens infected by Eimeria tenella during 6-12 days after infection.

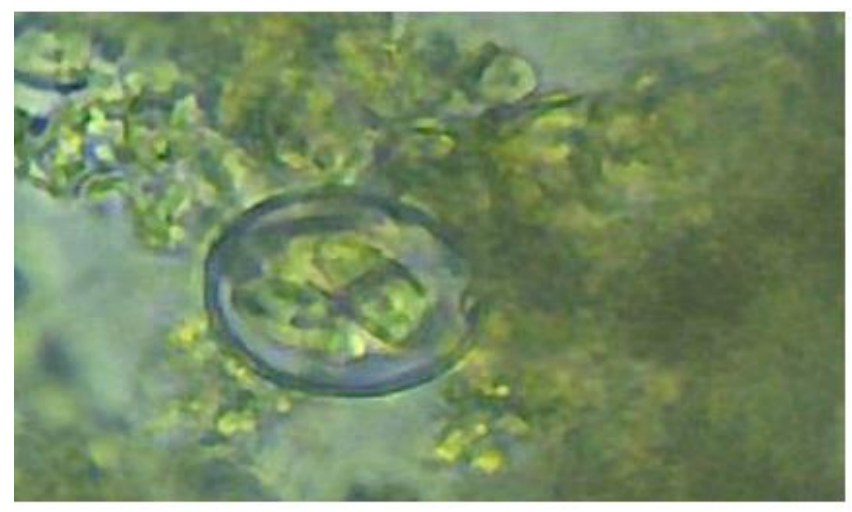

Figure 3. Eimeria tenella not immersed in formalin in feces sample of infected broiler chicken.

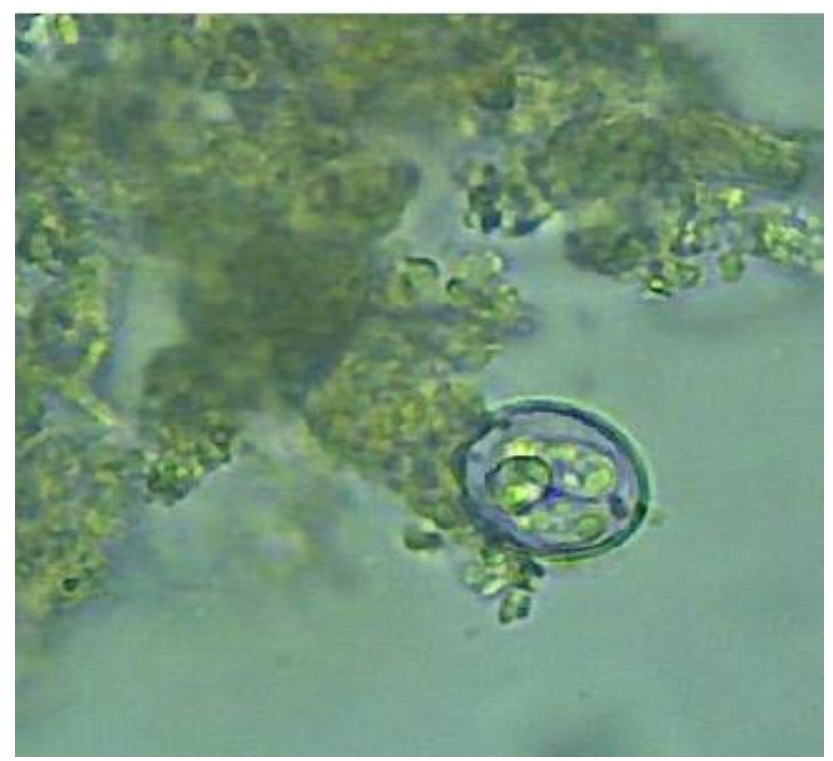

Figure 4. Eimeria tenella immersed in formalin with $0.6 \%$ concentration in feces sample of infected broiler chicken.

\section{DISCUSSION}

The analyzed data from oocytes production demonstrated significant differences among the chickens in the control group inoculated with $E$. tenella oocysts which were not immersed in formalin and the treatment groups that inoculated with $E$. tenella oocysts immersed in formalin. Formalin reacts with amino and amide groups in proteins, as well as with amino bound to non-water substances with the basic ingredients of purine nucleic acids and pyrimidines to form cross-bonds. Hence, it results in $E$. tenella structural rigidity, and consequently reduced pathogenicity. Although E. tenella is alive, it cannot inflict any disease and it still has significant influences on forming antibodies (Tizard, 2004).

There are several vital factors affecting the pathogenicity of E. tenella infection as follows, the number of swallowed oocysts, oocyst strains, environmental factors, the number of formed merozoites, and the amount of merozoites production in each cycle of schizogony. These cycles commence from the damage in host epithelial cells, the parasites settling in the tissue and cells of the host, the rate of re-infecting, and the level of immunity. Young animals are more sensitive to this disease than older ones. However, it generally does not cause death (Cui et al., 2016).

The most pathogenic stage in E. tenella life cycle is the second schizont generation which is large and contains hundreds of merozoites. The schizont develops in the lamina propria so that the mucosa will experience necrosis 
when the schizont grows and releases merozoites. Generally, death occurs five and six days after infection. In acute infections, death is possible to occur within a few hours after initial clinical symptoms arise (Srinivasu et al., 2019).

The attenuation of E. tenella can be conducted using formaldehyde (formalin) chemicals. Formaldehyde reacts with amino and amide groups in proteins, as well as with amino bound to non-water substances with the necessary ingredients of purine nucleic acids and pyrimidines to form cross-bonds. It, thus, provides structural rigidity in the organism. The structural rigidity of the organism can decrease organism virulence. As a result, the organism is alive, however, it cannot cause any disease. It significantly influences the antibodies forming (Tizard, 2004).

The coccidiosis can be acute or fatal, depending on the dose of infective oocysts swallowed by the host. In an infection with less than 150 oocysts, there will be bleeding points on the mucosa of the cecum with slight discoloration of the cecum wall. The number of 150-500 oocysts may cause bleeding in the mucosa with little bleeding, injury, and thickening of the cecum wall. Meanwhile, 1,000-3,000 oocysts cause high mortality, bleeding, some blood clots, and abnormal cecum contents. In addition, 5,000 or more oocysts will lead to the highest rate of death and bleeding, blood clots in the cecum, which are frozen and calcified (Srinivasu et al., 2019).

The clinical symptoms in coccidiosis are characterized by mucoid and hemorrhagic diarrhea. The symptoms of diarrhea are usually followed by dry feather, dehydration, anemia, lethargy, bending of the head and neck, and drowsiness. The chicken begins to excrete bloody feces 96 hours post-infection. The bleeding reaches its summit five or six days post-infection and in a severe case, it can be followed by death. In case an animal survives, the disease can be healed and the animal gets immune after seven days. In acute events, death occurs four to five days after infection (Boulton et al., 2018; Fortuoso et al., 2019).

Formaldehyde functions to denature protein enzymes which cause the alteration of the enzyme structure. Therefore, the enzyme activity is inhibited resulting in an inability to catalyze the metabolic processes in cells, and consequently microorganisms' death. Formalin can chemically react with almost all substances in the body cells to suppress cell function and cause cell death (Sarot et al., 2017). Formalin aldehydes are easy to react with proteins (Musiał et al., 2016). Formalin can react with $E$. tenella oocysts. This is because the outer layer of the $E$. tenella oocyst wall consists of protein, meanwhile, the inner layer consists of fat which binds to protein. Formalin will bind to proteins and cause protein death in the $E$. tenella oocyst wall. Formalin can dehydrate the cell of an organism (lack of water), consequently, the organism will dry up and structural rigidity occurs (Fortuoso et al., 2019).

Formalin concentration levels that are effective in attenuating $E$. tenella can cause low oocyst production. $E$. tenella immersion in $0.3 \%$ formalin can significantly affect antibody response of the host against Eimeria infection and protects chickens against severe cecum histopathological changes (Kadhim, 2014).

\section{CONCLUSION}

Formalin could attenuate the Eimeria tenella pathogenicity in broiler chickens. The difference in formalin concentration affected the amount of Eimeria tenella oocyst production in broiler chickens. In addition, a concentration of $1.2 \%$ formalin was the most optimal dose of formalin for Eimeria tenella attenuation.

\section{REFERENCES}

Ayed LB, Yang W, Widmer G, Cama V, Ortega Y, and Xiao L (2012). Survey and genetic characterization of wastewater in tunisia for Cryptosporidium spp., giardia duodenalis, enterocytozoon bieneusi, cyclospora cayetanensis, and Eimeria spp. Journal of Water and Health, 10(3): 431-444. DOI: https://doi.org/10.2166/wh.2012.204

Bahrami AM, and Bahrami A (2006). Immune response of chicken to an experimental sonicated coccidia oocyst vaccine. Archives of Razil Institutes, 61(1): 43-48. DOI: https://dx.doi.org/10.22092/ari.2006.103726

Boulton K, Nolan MJ, Wu Z, Psifidi A, Riggio V, Harman K, Boulton K, Nolan MJ, Wu Z, Psifidi A et al. (2018). Phenotypic and genetic variation in the response of chickens to Eimeria Tenella induced coccidiosis. Genetics Selection Evolution, 50(1): Article code 63. DOI: https://doi.org/10.1186/s12711-018-0433-7

Cui N, Wang Q, Shi W, Han L, Wang J, Ma X, Li H, Fangkun Wang F, Su S, and Zhao X (2016). Synergy of subgroup svian leukosis virus and Eimeria Tenella to increase pathogenesis in specific-pathogen-free chickens. Veterinary Immunology and Immunopathology, 177: 4247. DOI: https://doi.org/10.1016/j.vetimm.2016.06.001

Dalloul RA, and Lillehoj HS (2006). Poultry coccidiosis: Recent advancements in control measures and vaccine development. Expert Review of Vaccines, 5(1): 143-163. DOI: https://doi.org/10.1586/14760584.5.1.143

Fanatico AC, Pillai PB, Emmert JL, Gbur EE, Meullenet JF, and Owens CM (2007). Sensory attributes of slow and fastgrowing chicken genotypes raised indoors or with outdoor access. Poultry Science, 86(11): 2441-2449. DOI: 
https://doi.org/10.3382/ps.2007-00092

Fortuoso BF, Dos Reis JH, Gebert RR, Barreta M, Griss LG, Casagrande RA, Cristo TGD, Fabio Santiani F, Campigotto G, Rampazzo L et al. (2019). Glycerol monolaurate in the diet of broiler chickens replacing conventional antimicrobials: Impact on health, performance, and meat quality. Microbe Pathogen, 129: 161-167. https://doi.org/10.1016/j.micpath.2019.02.005

Gussem MD (2007). Coccidiosis in poultry: review on diagnosis, control, prevention and interaction with overall gut health. 16th European Symposium on Poultry Nutrition, pp. 253261. Available

at: file:///C:/Users/cyber/Downloads/Coccidiosis_in_poultry_ $\underline{\text { Review on diagnosis control.pdf }}$

Hafex H (2011). Enteric diseases of poultry with special attention to clostridium perfringens. Pakistan Veterinary Journal, 31(3): 175-184. Available at: file:///C:/Users/cyber/Downloads/Enteric_Diseases_of_Po ultry_with_Special_Attention.pdf

Jordan A, Caldwell DJ, Klein J, Coppedge J, Pohl S, Fitz-Coy S, and Lee JT (2011). Eimeria tenella oocyst shedding and output in cecal or fecal contents following experimental challenge in broilers. Poultry science, 90(5): 990-995. Doi: https://doi.org/10.3382/ps.2010-01228

Kadhim LI (2014). Histopathological changes of broilers immunized with sonicated oocysts against Eimerria Tenella. International Journal of Advanced Biologocal
Research, 4(1): 31-35. available at: http://scienceandnature.org/IJABR/IJABR_Vol4(1)2014/IJ ABR_V4(1)14-8.pdf

Meeusen ENT, Walker J, Peters A, Pastoret PP, and Jungersen G (2007). Current status of veterinary vaccines. Clinical Microbiology Review, 20(3): 489-510. Available at: https://cmr.asm.org/content/20/3/489

Musiał A, Gryglewski RW, Kielczewski S, Loukas M, and Wajda J (2016). Formalin use in anatomical and histological science in the 19th and 20th centuries. Folia Medica Cracoviensia, 56(3): 31-40. Available at: http://www.fmc.cm-uj.krakow.pl/pdf/56_3_31.pdf

Sarot E, Carillo-Baraglioli MF, Duranthon F, Pequignot A, and Pyronnet S (2017). Assessment of alternatives to environmental toxic formalin for DNA Conservation in biological specimens. Environmental Science and Pollution Research, 24(20): 16985-16993. DOI: https://doi.org/10.1007/s11356-017-9349-y

Srinivasu B, Preetam VC, and Srinivas G (2019). Effect of cocciban herbal coccidiostats on hemato biochemical, fecal parameters and cecal histopathology of broiler chicken. Tropical Animal Health and Production, 51(6): 1375-1381. DOI: https://doi.org/10.1007/s11250-019-01831-3

Tizard (2004). Veterinary Immunology: An Introduction. 7th Ed. USA: Saunders, pp. 207-211. Available at: https://www.amazon.com/Veterinary-ImmunologyIntroduction-Saunders-Paperback/dp/B00M3UQJ0A 